\title{
Mykosen und Chlamydien in Gynäkologie und Geburtshilfe
}

\author{
Werner Mendling, Monika Hampl
}

\section{Übersicht}

Mykosen

Chlamydien

\section{Mykosen}

\section{Einführung}

Obwohl die Vulvovaginalkandidose eine gelegentliche und eher banale Erkrankung der jungen Frau zu sein scheint, kann sie schwierig zu diagnostizieren sein und im Fall von chronischen Rezidiven für die Patientin und die Ärzte zur Qual werden. Darüber hinaus werden heute mehr als $80 \%$ aller (vermuteten) Vulvovaginalkandidosen ohne ärztliche Diagnose von der Patientin durch Kauf eines Antimykotikums mit wahrscheinlich hoher Quote von Fehldiagnosen und suboptimalen Therapieformen selbst behandelt. Mit diesem Artikel soll der aktuelle Wissensstand dargestellt werden.

In der Frauenheilkunde werden Mykosen fast immer von Candida-Arten verursacht, während zoophile Dermatophytosen (z.B. Trichophytie, Mikrosporie) von Meerschweinchen oder Katzen gerade bei Kindern und Jugendlichen, die gern mit den Tieren schmusen, auch auf der Haut des Bauch- oder Genitalbereichs vorkommen können [1].

Die Vulvovaginalkandidose ist eine Infektion der östrogenisierten Vagina und des Vestibulums mit gelegentlicher Ausdehnung auf die gesamte Vulva, Perianalund Leistenregion.
Eine Vaginalkandidose tritt nicht vor der Menarche oder postmenopausal auf!

\section{Tipp für die Praxis}

Es sollte die Bezeichnung „Vulvovaginalkandidose“ (VVK) oder „Candida-albicans-Vulvovaginitis“ bevorzugt werden. Die Endung „-iasis“ sollte parasitären Infektionen vorbehalten bleiben, z. B. Trichomoniasis, sie wird aber wegen der weiten Verbreitung im angloamerikanischen Schrifttum leider häufig benutzt [2].

Bei mindestens 4 Erkrankungen pro Jahr sprechen wir von einer chronisch rezidivierenden Vulvovaginalkandidose (CRVVK), die bei etwa 6-9\% der Frauen mit VVK auftritt [3].

\section{Mikrobiologie}

85-95\% der die Vagina kolonisierenden Candida-Spezies bei adoleszenten, prämenopausalen oder schwangeren, asymptomatischen, gesunden Frauen und bei Frauen mit akuter VVK sind Candida albicans (s. Tab. 1). Hingegen werden Non-albicans-Arten, besonders Candida glabrata, häufiger bei postmenopausalen, bei diabetischen und bei immunsupprimierten Frauen und Mädchen gefunden [2]. 


\section{Tabelle 1}

Verteilung der Candida-Arten bei $\mathbf{4 7 2}$ prämenopausalen Frauen mit akuter Vaginalkandidose in Polen und Deutschland (aus [2]).

\section{akute Candida-Vulvovaginitis}

n

472

450

10

4

C. krusei

andere (C. tropicalis, C. kefyr, C. africana, S. cerevisiae)
$\%$ 100

95,3

0,9

2,3

\section{Risikofaktoren einer VVK}

bzw. von Rezidiven

- Diabetes mellitus

- Allergien (z. B. Neurodermitis, Typ-I-Allergien)

- Sexualverkehr und Orogenitalkontakte

- genetische Prädisposition

- psychosozialer Stress

Genitale Kolonisation. Etwa 30\% der prämenopausalen Frauen (kulturell gemessen, mit PCR gemessen über $50 \%$ ) sind vaginal asymptomatisch von Candida kolonisiert.

Prädisponierende Wirtsfaktoren. Patientinnen mit Diabetes mellitus leiden häufiger unter einer VVK, und die Therapie versagt, solange die Serumglukosespiegel nicht normalisiert sind [4].

Die vaginale Candida-Kolonisation wird von oralen Kontrazeptiva mit modernen niedrigen Östrogenspiegeln vermutlich nicht erhöht [2].

Nach Sexualverkehr und besonders Orogenitalkontakten werden gehäuft Re-Infekte beobachtet $[5,6])$. Nicht zuletzt sind auch genetische Faktoren für Rückfälle verantwortlich. Bei der Candida-Abwehr ist aber auch das vaginale Mikrobiom bedeutsam.

Mädchen und junge Frauen mit Atopien bzw. Neurodermitis und Typ-I-Allergien entwickeln signifikant häufiger eine VVK als andere [7]. Die klinischen Zeichen der VVK, wie Rötung und Juckreiz, werden besonders bei Rezidiven als Ausdruck allergischer Phänomene gesehen [8]. Auch psychosozialer Stress kann eine VVK auslösen [9].

\section{Neonatale Kandidosen}

Gesunde reife Neugeborene, die während der vaginalen Geburt von der mütterlichen Vagina mit Candida albicans kolonisiert worden sind (rechnerisch $10-20 \%$ je nach Sectioquote), erleiden häufig während des 1 . Lebensjahrs mit einem Gipfel in der 2.-4. Lebenswoche eine Mund- und/oder Anogenitalkandidose („Mundsoor“, „Windeldermatitis“) [10]. Dabei führt die Kolonisation wegen der immunologischen Inkompetenz der Säuglinge zur Infektion. In Deutschland gibt es deshalb eine Empfehlung zur prophylaktischen Therapie der asymptomatischen Candida-Kolonisation in den letzten Wochen der Schwangerschaft. Dadurch wird die Frequenz der Candida-Besiedlung des Säuglings signifikant reduziert [10]. Leider fehlen zu der Problematik seit über 30 Jahren weltweit moderne Studien!

\section{Häufigkeit von akuten Vulvovaginalkandidosen}

Aufgrund fehlender Östrogene kommt präpubertär eine VVK praktisch nicht vor [11].

In Stockholm wurde die Frequenz von Candida-Kolonisation bzw. -Infektion bei 219 sexuell aktiven adoleszenten Mädchen/Frauen in Abhängigkeit vom Sexualverhalten untersucht [6]: 128 (60\%) hatten wenigstens einmal eine VVK, davon 45 (22\%) mehr als 3-mal, bei 19 (9\%) wurde Chlamydia trachomatis gefunden und bei 65 (32\%) ein unangenehmer Geruch des Fluors als Zeichen einer bakteriellen Vaginose. Der Nachweis von Candida in der Vagina bzw. VVK war signifikant korreliert mit Orogenitalverkehr (50\%, p = 0,002, Odds Ratio $1,97)$ und dem Verzehr von Süßigkeiten mindestens 3-mal pro Woche (24\%, p = 0,06). Von den 93 Mädchen und Frauen mit vaginalem Candida-Nachweis waren 79 (85\%) typisch symptomatisch. Frauen, die orale Kontrazeptiva mindestens seit 3 Jahren eingenommen hatten (31\%), litten doppelt so oft unter einer CRVVK als solche mit kürzerer Einnahmezeit (14\%).

\section{Klinische Symptomatik}

Entsprechend des Östrogeneinflusses leiden Jugendliche und junge Frauen gewöhnlich primär unter einer Vaginalkandidose, die sich auch auf die (innere und äußere) Vulva ausdehnen kann. Bei Mädchen vor der Menarche und postmenopausalen Frauen manifestiert sich die Infektion hingegen primär als Vulva- und/oder Interkruralkandidose. 
Die klinischen Zeichen treten typischerweise prämenstruell auf: Die von Östrogenen induzierte Zellproliferation und von Progesteron induzierte Zytolyse setzt Glykogen frei, das von Laktobazillen verstoffwechselt werden kann, sodass der Zuckerspiegel in der Vagina erhöht ist, was wiederum das Candida-Wachstum fördert.

In etwa $90 \%$ ist der Juckreiz das wichtigste, jedoch keinesfalls verlässliche Symptom, da in nur 35-40\% der Fälle mit genitalem Juckreiz eine VVK vorliegt $[12,13]$.

Der Ausfluss kann sehr unterschiedlich und oft zu Beginn einer akuten VVK dünnflüssig, später flockig sein.

\section{Bei der CRVVK kann der Ausfluss oft gänzlich fehlen.}

Die meisten Patientinnen beklagen vaginale Rötung, Wundheitsgefühl, Brennen und Dysurie, bei Rezidiven und bei Atopikerinnen oft auch Rhagaden. Bei schweren Fällen kann eine dicke Fluorschicht an der Vaginalwand adhärent sein, die beim Abwischen zu kleinen Blutungen führen kann.

Aus dermatologischer Sicht wird die Vulvakandidose differenziert in eine

- vesikulöse,

- exzematoide (Abb. $\mathbf{1}$ ) oder

- follikuläre Form [2].

Infektion mit anderen Candida-Arten. Die Candidaglabrata-Vaginitis ist trotz häufiger Kolonisation selten und kommt gewöhnlich in der späteren prä- und perimenopausalen Zeit vor $[2,14]$. Die Candida-kruseiVaginitis, die Candida-parapsilosis-Vaginitis und als Rarität die Saccharomyces-cerevisiae-Vaginitis sind meist ähnlich wie die Candida-glabrata-Vaginitis mit nur milden klinischen Symptomen und Beschwerden verbunden (s.Tab. 1).

Sonstige Infektionen. Differenzialdiagnostisch kommen bei Kindern eine begleitende Vulvovaginitis bei Masern, eine A-Streptokokken-Vulvovaginitis, ansonsten Herpes genitalis, atopische Vulvitiden oder bakterielle Infektionen der weniger widerstandsfähigen Introitushaut in der hormonalen Ruhephase oder selten heute die Trichomoniasis in Betracht.

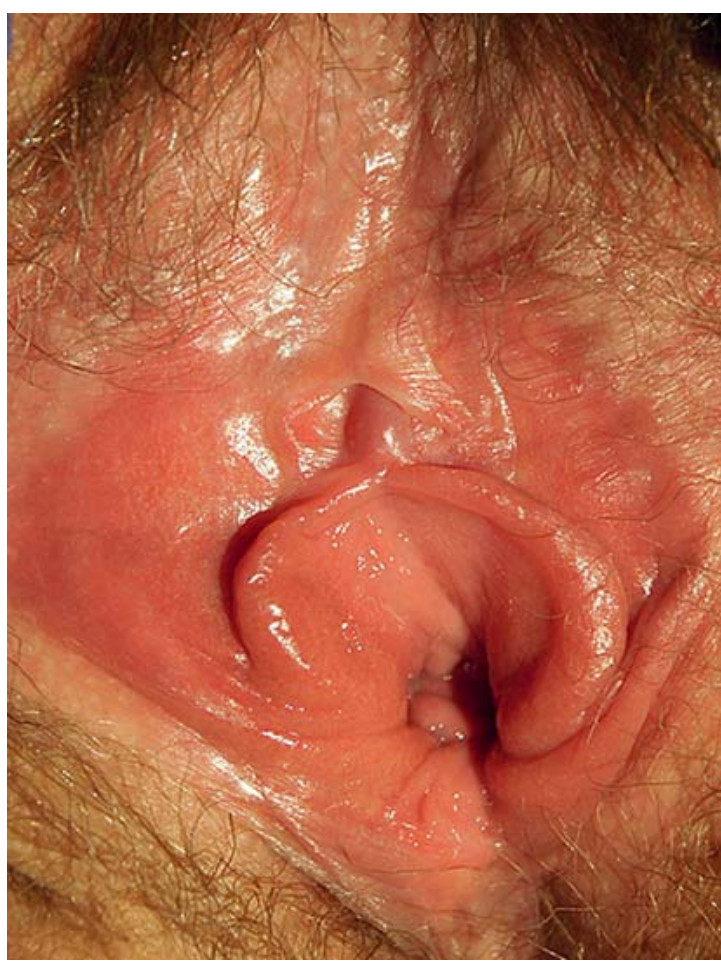

Abb. 1 Ekzematoide Vulvovaginalkandidose durch Candida albicans.

\section{Tipp für die Praxis}

Häufig wird die bei Berührung/Penetration provozierte Vestibulodynie, bei der stechender Schmerz eine Untersuchung unerträglich machen kann, von den Frauen selbst, aber auch von klinisch unerfahrenen Ärzten mit Antimykotika „malträtiert“.

\section{Diagnostik}

Die Diagnose der VVK wird immer durch die Kombination von Anamnese, den klinischen Zeichen und durch den Nachweis von Hefepilzen gestellt. Die klinische Diagnose kann schwierig sein, denn trotz CandidaNachweis, bei z. B. Juckreiz im Introitus, muss nicht unbedingt eine VVK vorliegen.

Notwendige Diagnostik. Essenziell sind die Anamnese, die gynäkologische Untersuchung und die mikroskopische Untersuchung des Fluors mit Kochsalzlösung oder 10\%iger KOH-Lösung bei 400-facher Vergrößerung im Licht- bzw. besser Phasenkontrastmikroskop $[2,15]$. Gegebenenfalls erfolgt auch eine pH-Messung. Sprosszellen (Blastosporen) oder (Pseudo-)Hyphen/Myzelien können in etwa $50-80 \%$ der Fälle von Vaginalkandidosen im Mikroskop gefunden werden [5]. Es können, müssen aber nicht, vermehrt Leukozyten im Fluor gefunden werden. 
Bei eindeutiger klinischer Symptomatik und sicherem mikroskopischen Candida-Nachweis wird derzeit aus pragmatischen und finanziellen Erwägungen die Pilzkultur mit Artbestimmung für entbehrlich gehalten. Falls mikroskopisch keine Blastosporen oder (Pseudo-) Hyphen gefunden werden können oder falls es sich um eine CRVVK oder einen komplizierten Fall handelt, ist eine kulturelle Untersuchung mit Artbestimmung notwendig [2].

\section{Tipp für die Praxis}

Eine In-vitro-Empfindlichkeitstestung ist nicht erforderlich, allenfalls bei Nachweis von Non-Candida-albicans-Arten und chronisch rezidivierenden Verläufen. Das sollte aber in einem mykologisch versierten Labor erfolgen. Häufig kommt es nämlich vor, dass „Resistenzen" gegen Antimykotika resultieren, die in Wirklichkeit nicht bestehen.

Unsinnige Diagnostik. Serologische Tests werden als nicht nützlich für die Diagnose der VVK angesehen, weil bei den meisten Menschen schwache Antikörpertiter vorliegen und VVK nicht zu einer Titerveränderung führen.

\section{Therapie}

Es stehen Medikamente aus verschiedenen Wirkstoffgruppen zur Verfügung. So bilden Polyene Komplexe mit dem Ergosterol der Sprosspilzmembran und verändern dadurch deren Permeabilität. Azole verhindern die Bildung von Lanosterol zu Ergosterol in der Hefezellmembran. Und Ciclopiroxolamin behindert durch Chelatbildung wichtige Enzyme, die Eisen benötigen.

Kolonisation. Die asymptomatische vaginale Kolonisation bedarf selbst bei hoher Keimzahl keiner Therapie, falls die Patientin immunkompetent ist und unter keiner CRVVK leidet.

In retrospektiven und in einer prospektiv randomisierten Studie wurde aber überraschend eine signifikante Reduktion von Frühgeburten nach vaginaler Behandlung mit Clotrimazol im 1. Trimester der Schwangerschaft festgestellt $[16,17]$.

Seit Einführung der Triazole um 1990 wurde keine neonatale Teratogenität nach Einnahme im 1. Trimenon beobachtet.
Therapie der akuten Vulvovaginalkandidose. Die akute VVK kann lokal behandelt werden mit:

- Polyenen (Nystatin, Amphotericin B)

- Imidazolen (z. B. Clotrimazol, Miconazol-Nitrat, Econazol-Nitrat, Fenticonazol-Nitrat)

- Ciclopiroxolamin

Es gibt Vaginalsuppositorien oder Vaginalcremes mit Dosierungen und Zubereitungen für Behandlungsdauern, die bei 1 -3 oder 6-7 Tagen liegen. Es ist aber auch die orale Behandlung mit den Triazolen Fluconazol $1 \times 150 \mathrm{mg}$ (oder Itraconazol $2 \times 200 \mathrm{mg}$ ) möglich; Nystatin wird bei oraler Einnahme nicht resorbiert.

Alle mykologischen und klinischen Heilungsergebnisse nach den verschiedenen geprüften Therapieverfahren sind außerhalb der Schwangerschaft gleich gut und bewegen sich zwischen 85\% 1-2 Wochen und 75\% 4-6 Wochen nach Behandlungsende [2].

Falls die Kandidose die Region der Vulva außerhalb des Introitus vaginae oder die Inguinalregion mit einschließt, ist eine antimykotische Hautcreme (z.B. Clotrimazol) für eine Behandlungsdauer von etwa 1 Woche $2 \times$ täglich empfehlenswert. Die kombinierte Behandlung der akuten VVK intravaginal und zusätzlich mit Creme für die Vulva scheint günstigere Heilungsergebnisse zu erzielen als die intravaginale Therapie alleine [18].

Die „blinde“ Behandlung des asymptomatischen Sexualpartners ist für die Patientin ohne Nutzen.

Resistenz von Candida albicans? Obwohl vaginale Candida-albicans-Stämme mit höheren minimalen Hemmkonzentrationen gegen Fluconazol gefunden wurden, sind Fälle von Azol-Resistenz in der Gynäkologie eine Rarität [5].

Klinische Resistenz korreliert nicht mit minimalen Hemmkonzentrationen und umgekehrt. Deshalb werden Resistenztests üblicherweise in der Gynäkologie nicht empfohlen [2,5], es sei denn, es handelt sich um Rezidive mit Non-Candida-albicans-Arten.

Non-Candida-albicans-Vaginitis. Die übliche vaginale oder orale Behandlung versagt gewöhnlich bei der Candida-glabrata-Vaginitis. Diese ist aber selten, denn meist handelt es sich nur um Kolonisationen! Wegen der Selektion gegen Fluconazol resistente Stämme wird neuerdings die 14-tägige Therapie mit intravenösem Micafungin diskutiert, einem Echinocandin, das für 


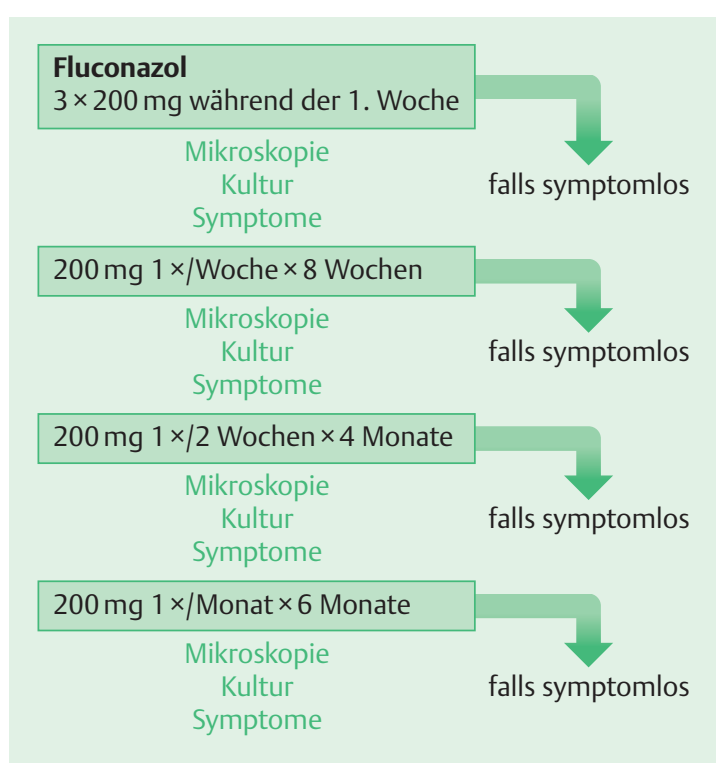

Abb. 2 Individualisierte, dosisreduzierende Erhaltungstherapie mit Fluconazol bei chronisch rezidivierender Vulvovaginalkandidose (modifiziert nach [20]).

lebensbedrohliche Mykosen, z. B. in der Hämato-Onkologie, zugelassen und sehr teuer ist [19].

Die Candida-krusei-Vaginitis ist gegen Fluconazol und Flucytosin resistent, jedoch können lokal Clotrimazol, Ciclopiroxolamin oder Nystatin angewendet werden $[12,19]$.

Chronisch rezidivierende VVK. Da Infektion gleich Kolonisation plus Disposition ist und eine Therapie gegen die Disposition (die immunologische lokale Schwäche) bisher nicht erprobt ist, werden bei der CRVVK lokale oder orale Erhaltungstherapien empfohlen, um Rückfälle zu vermeiden.

Bei der Hälfte der Patientinnen tritt ein Rückfall bereits kurz nach Beendigung der Therapie auf.

Deshalb ist die von Donders et al. (2008) empfohlene Therapie bzw. Prophylaxe mit einer Initialdosis von $3 \times 200 \mathrm{mg}$ Fluconazol in der 1 . Woche, gefolgt von einem die Dosis reduzierenden Erhaltungsregime ein Fortschritt, weil in seiner Studie fast 90\% der Patientinnen nach 6 Monaten und 77\% der Patientinnen nach 1 Jahr krankheitsfrei waren (Abb. 2) [20]. Die kumulative Gesamtdosis Fluconazol ist bei dieser Therapie geringer als bei vergleichbar anderen bzw. wesentlich höheren Therapieregimes gegen Organmykosen.

\section{Alternativen zu Antimykotika?}

Die um 1970 von Prof. Hans Rieth, einem der bedeutendsten deutschen Mykologen, propagierte „Anti-PilzDiät“ (ballaststoffreiche und zuckerarme Ernährung) ist zur Therapie von CRVVK seit etwa 20 Jahren mangels Evidenz verlassen worden.

Ansätze zu einer immunologischen Therapie. Es gibt bis jetzt noch keine zufriedenstellende Immuntherapie für die CRVVK. Rusch und Schwiertz berichten 2006 über erste Ergebnisse mit einer Candida-Autovakzination, die aber nur die allergoide Komponente im Sinn einer Desensibilisierung betrifft [21]. Mindestens 2 Impfstoffe sind in Diskussion mit der Hoffnung auf klinische Wirksamkeit, die nach Boosterung anhält [22].

Bedeutung von Laktobazillen. Die intramuskuläre Injektion von nicht $\mathrm{H}_{2} \mathrm{O}_{2}$-bildenden Laktobazillen, die eine unspezifische Immunstimulation und die Bildung von IgA-Antikörpern zur Folge hat, kann die Häufigkeit von CRVVK nicht reduzieren [23]. Es sind aber Laktobazillusstämme identifiziert, die in vitro einen fungiziden bzw. immunstimulierenden Effekt haben, sowie andere, die in vivo nach Therapie einer VVK die vaginale Kolonisation gegen Placebo signifikant verringern [24]. Andererseits hat die monatliche Zugabe von Laktobazillen für je 6 Tage zu Itraconazol $2 \times 200 \mathrm{mg} / \mathrm{d}$ gegenüber Itraconazol allein über 6 Monate keine Verbesserung der Rezidivrate von CRVVK ergeben. Diese Maßnahmen waren aber hochsignifikant besser als klassische Homöopathie [25].

\section{Selbstmedikation (OTC-Therapie)}

Die Selbsttherapie („Over-the-Counter“ = OTC) der VVK mit Clotrimazol, und in einigen Ländern auch mit Fluconazol, wird mittlerweile in weit mehr als $80 \%$ der Fälle betrieben. Sie ist aber bei Mädchen/Frauen unter 18 Jahren in Deutschland nicht gestattet. Obendrein ist die Selbsteinschätzung der Patientin sehr unzuverlässig [26]. 


\section{Chlamydien}

\section{Biologische Grundlagen}

Erscheinungsformen. Chlamydien sind sehr kleine, primitive Bakterien mit einer starren Zellwand aus Lipopolysacchariden. Das Genom ist mit ca. 600 Genen klein, was dazu führt, dass Chlamydien keinen eigenen Energiestoffwechsel haben, kein ATP bilden und somit nicht selbstständig leben können, sondern auf den Stoffwechsel anderer Zellen zur Vermehrung angewiesen sind (obligat intrazelluläre Erreger).

Chlamydien kommen als Elementarkörperchen und als Retikularkörperchen vor.

Die Elementarkörperchen der Chlamydien stellen die infektiöse Form dar. Sie liegen in Einschlusskörperchen in der Zelle vor, haben nur einen geringen Stoffwechselumsatz und sind daher in dieser Phase resistent gegen Antibiotika. In der Vermehrungsphase entstehen Retikularkörperchen. In dieser Phase kann die Vermehrung der Bakterien gut mit Antibiotika gehemmt werden.

Vermehrungszyklus. Ein vollständiger Vermehrungszyklus von Chlamydien in einer Wirtszelle dauert 36-50 Stunden, dabei wird die Wirtszelle zerstört, und die Elementarkörperchen kommen aus den Einschlusskörperchen frei. Diese können außerhalb der Zelle überleben und sind somit die eigentlichen infektiösen Partikel, die weitere Zellen infizieren. Durch den Zerfall der Wirtszellen entstehen Ulzera, es kommt zur Zytokinausschüttung mit Entzündungsreaktion und zur Nekrose der Schleimhaut. Die Einwanderung von Granulozyten und Lymphozyten bedingt Eiterentstehung. Jede Chlamydien-Infektion löst die Bildung von Antikörpern aus: Es entstehen lokale IgA- und systemische IgM/IgG-Antikörper. Diese humorale Immunantwort führt jedoch nicht zu einer belastbaren protektiven Immunität, denn chronische Infektionen sind häufig. Auch Infektionen mit anderen Serotypen können durch diese Immunantwort nicht verhindert werden.

\section{Tipp für die Praxis}

Eine akute Infektion mit Chlamydien kann am besten therapiert und auch ausgeheilt werden, sobald die Infektion in ein chronisches Stadium übergeht. Denn wenn in vielen Zellen die Chlamydien in der Ruhephase vorliegen, können Antibiotika schlechter angreifen.

\section{Infektionswege}

Die Übertragung der Chlamydien ist bei den verschiedenen Serotypen unterschiedlich:

- C. trachomatis der Serogruppe A-C, verantwortlich für das Trachom am Auge, wird durch Schmierinfektion übertragen, z.B. direkter körperlicher Kontakt, kontaminierte Gegenstände (z.B. Augenbrauenstift) oder verschmutzte Handtücher.

- C. trachomatis der Serogruppe D-K werden hauptsächlich durch sexuellen Kontakt übertragen oder peripartal von der Mutter auf Neugeborene. Die Chlamydien befallen bevorzugt hochzylindrische Schleimhautepithelzellen (z. B. Zervix, Urethra, Konjunktiva). Die Plattenepithelien der Vagina und Vulva können nicht infiziert werden.

- Die Serotypen L1 -L3 bedingen das Lymphogranuloma venereum und gehören zu den Erregern von einer der 4 Geschlechtskrankheiten.

- C. pneumoniae wird durch Tröpfcheninfektion übertragen und verursacht milde respiratorische Infekte.

- C. psittaci findet sich v. a. bei Vögeln, die das Hauptreservoir bilden. Dieses Bakterium wird von Tier zu Tier, aber auch in seltenen Fällen vom Tier auf den Menschen oder von Mensch zu Mensch übertragen. Gefährdet sind Menschen, die in der Geflügelzucht arbeiten oder Vögel als Heimtiere halten.

\section{Krankheitsbilder abhängig von den Serotypen}

\section{C. trachomatis Serotyp A-C}

Das Trachom ist eine chronische Augenentzündung in Form einer Follikulitis, die durch Chlamydia trachomatis der Serogruppe A -C hervorgerufen wird. Es handelt sich um eine Schmierinfektion. Unbehandelt führt diese Infektion nach 10-30 Jahren zur Erblindung durch Ausdehnung der Entzündung auf die Kornea mit Ulzerationen und Narben. Die Erkrankung betrifft v.a. Menschen in Endemiegebieten in trockenen, heißen Zonen mit Wassermangel, z.B. in Afrika.

\section{C. trachomatis Serotyp $L 1-L 3$ \\ (Lymphogranuloma venereum)}

Die Erreger des Lymphogranuloma venerum sind deutlich virulenter als andere Chlamydien-Stämme. Diese klassische Geschlechtskrankheit verläuft in 2 Stadien. Nach Geschlechtsverkehr mit Übertragung der Bakterien entsteht nach 3-21 Tagen ein kleines Ulkus oder eine schmerzlose Papel im Genitale (Scheide, Zervix, 


\begin{tabular}{|l|l|}
\hline Tabelle 2 \\
\hline Therapie der Chlamydien-Infektion [27]. \\
\hline Präparat & Dosierung \\
\hline 1. Wahl & $200 \mathrm{mg} /$ Tag über 7 Tage \\
\hline Doxycyclin & $4 \times 500 \mathrm{mg} /$ Tag \\
\hline Tetrazykline & \\
\hline 2. Wahl & $4 \times 500 \mathrm{mg} /$ Tag \\
\hline Erythromycin & $300 \mathrm{mg} / \mathrm{d}$ \\
\hline Roxythromycin & Einmaltherapie $1 \mathrm{~g}$ oder $2 \times 1 \mathrm{~g}$ \\
\hline Azithromycin & \\
\hline 3. Wahl & $1 \times 400 \mathrm{mg} / \mathrm{d}$ \\
\hline Ofloxacin & $2 \times 750 \mathrm{mg} / \mathrm{d}$ \\
\hline Ciprofloxacin & \\
\hline Moxifloxacin & \\
\hline Amoxicillin & \\
\hline
\end{tabular}

Vulva, anorektal), die rasch abheilt. Erst nach Tagen bis Wochen kommt es zur Anschwellung der regionären Lymphknoten (kleines Becken, inguinal, perirektal etc.), die einschmelzen und abszedieren können, aufbrechen und dann mit derben Narben abheilen. Entsprechende Folgen sind Lymphangitis, Ödeme, Elephantiasis der Beine. Rezidive sind häufig durch Fistelungen in den eingeschmolzenen Lymphknoten. Die Therapie erfolgt mit Antibiotika über 2-4 Wochen (s. Tab. 2).

\section{C. pneumoniae}

C. pneumoniae sind die bei uns am weitesten verbreiteten Chlamydien mit einer Durchseuchung (typenspezifischem Antikörpernachweis) von 50-70\% im Erwachsenenalter. Die Bakterien werden durch Tröpfcheninfektion übertragen und verursachen leichte respiratorische Infekte. Problematisch sind diese Infek-

\section{Tabelle 3}

Krankheitsbilder durch Chlamydia trachomatis der Serogruppe D-K.

\begin{tabular}{ll}
\hline Frau & Mann \\
\hline - Urethritis & - Urethritis \\
- Zervizitis & - Prostatitis \\
- Endometritis & - Epididymitis \\
- Salpingitis & - Konjunktivitis \\
- Peritonitis & - Arthritis \\
- Perihepatitis & - Exanthem \\
- Konjunktivitis & - Morbus Reiter \\
- Arthritis & \\
- Exanthem & \\
- Morbus Reiter & \\
\hline
\end{tabular}

tionen durch die Folgeschäden an Knorpeln mit Arthritis und Atherosklerose von Gefäßen.

Die Therapie bei chlamydienbedingter chronischer Arthritis muss über 30-90 Tage erfolgen.

\section{C. psittaci}

Hauptreservoir sind Vögel. Eine Übertragung auf den Menschen ist selten, aber möglich. Die Infektion führt beim Menschen zur atypischen Pneumonie.

\section{C. trachomatis Serogruppe D-K}

Krankheitsbilder. C. trachomatis der Serogruppe D-K gehört zu den häufigsten sexuell übertragenen bakteriellen Erregern. Die Infektion ist häufig symptomarm oder symptomlos (in 90\% der Fälle) und bleibt deshalb lange unerkannt. Die infizierte Genitalschleimhaut dient als Reservoir für weitere Infektionen. Leider kann auch die klinisch inapparente Infektion zu postinfektiösen Schädigungen der Tuben und somit zu einer Sterilität führen. Bei einigen Individuen kann es zu akuten eitrigen Infektionen v.a. im Bereich der Adnexen mit Ausbreitung im kleinen Becken kommen (Pelveoperitonitis). Die Erkrankungen bei Mann und Frau sind in Tab. 3 aufgelistet. Die Infektion an sich ist nur in seltenen Fällen lebensbedrohlich, dagegen sind die Folgeschäden der Erkrankung weitreichend:

- Eileiterverschlüsse

- Extrauteringraviditäten

- chronische Schmerzsyndrome

- Arthritiden

- Kinderlosigkeit mit konsekutiver IVF-Behandlung 
Symptomatik. Die häufigste Infektion bei der Frau ist die Zervizitis mit gelblich, eitrigem Fluor, starker Gefäßzeichnung der Portiooberfläche, Kontaktblutungen und hyperplastischer Schleimhaut. Die Bakterien halten sich in der Regel an der Zervix nur einige Monate. Da sie mit den sich abschilfernden Zellen abgestoßen werden, kann es zu aufsteigenden Infektionen mit Endometritis (Symptom: Zwischenblutungen) und Adnexitis (Symptom: Unterbauchschmerzen) kommen. Die Bakterien können sich in der Adnexschleimhaut jahrelang halten. Dort kommt es zur chronischen Entzündung, die im fortgeschrittenen Stadium zum Funktionsverlust der Tube, Tubenverschluss, Verwachsungen und Pyosalpinx führen kann. Die Bakterien gelangen über die Tubenöffnung ins kleine Becken und über den Blutkreislauf bis in die Gelenke, wo sie durch Entzündung des Knorpels Arthritiden auslösen. Kommt es zur Peritonitis im kleinen Becken, kann es über peritoneale Aussaat zur Perihepatitis kommen. Auch eine Proktitis und Proktokolitis ist bei speziellen Sexualpraktiken möglich.

In bis zu $90 \%$ der Fälle verläuft die Infektion symptomlos.

Wegen des langen Vermehrungszyklus der Erreger entwickelt sich die Infektion eher langsam, bleibt deshalb oft lange symptomarm und unentdeckt und wird erst in einem Stadium diagnostiziert, wo sie bereits irreversible Folgeschäden angerichtet hat. Viele Frauen werden erst mit einer chronischen oder abgelaufenen Chlamydien-Infektion diagnostiziert, wenn es nicht zum spontanen Schwangerschaftseintritt kommt.

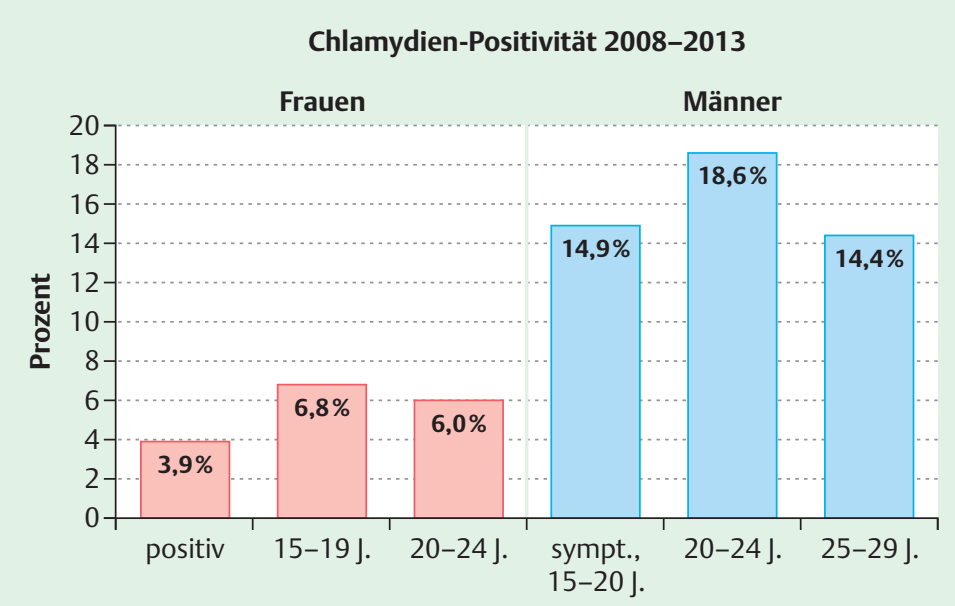

Abb. 3 Altersabhängige Chlamydien-Prävalenz in Deutschland 2008-2013; 21 Labore aus 14 Bundesländern [32].
Bei den Männern tritt die Chlamydien-Infektion in Form einer Urethritis in Erscheinung, aber auch hier verlaufen $90 \%$ der Infektionen unbemerkt. Es kann zur aszendierenden einseitigen Epididymitis mit der Folge einer Azoo- oder Oligospermie kommen.

Risiken. Die Prävalenz einer Infektion mit Chlamydia trachomatis wird in der Altersgruppe zwischen 20 und 39 Jahren in der Bundesrepublik mit 2,9\% (2,2-3,7\%) angegeben, wobei in der Altersgruppe zwischen 16 und 19 Jahren bei Frauen sowie 20 und 24 Jahren bei Männern die höchste Prävalenz für genitale Infektionen besteht (Abb. 3) [28]. Hier werden Prävalenzen von 4,4-4,9\% angegeben [29]. Unter Berücksichtigung dieser Zahlen ergeben sich 3 Risikokonstellationen, die im Folgenden erläutert werden sollen:

- Im Rahmen der ersten Risikokonstellation werden 3 Faktoren berücksichtigt: das Alter, die Anzahl der Sexualpartner sowie eine mögliche hormonelle Kontrazeption. Bei jungen Frauen (<25 Jahre) mit mehr als 5 Partnern innerhalb der letzten 5 Jahre ist eine 5-fach höhere Prävalenz zu erwarten. Bei Frauen mit hormoneller Kontrazeption steigt diese auf das 8-Fache. Die hormonelle Kontrazeption wird als Surrogatparameter betrachtet, da hier auf der einen Seite von einer sexuellen Aktivität ausgegangen werden kann, auf der anderen Seite die unter hormonellen Einflüssen häufiger beobachteten zervikalen Ektopien eine Prädisposition für eine C.-trachomatisInfektion darstellen.

- Die zweite Risikokonstellation berücksichtigt eine stattgehabte C.-trachomatis-Infektion innerhalb der letzten Monate. Dies erhöht die Prävalenz durch Reinfektion oder Persistenz auf 13,4\%.

- Schließlich ist eine deutlich erhöhte Prävalenz bei Patienten mit bereits diagnostizierten anderen STD (Sexually transmitted Diseases) anzunehmen, und umgekehrt stellt eine bekannte Chlamydien-Infektion ein erhöhtes Risiko für eine HIV- oder HPVInfektion dar [30,31].

Diagnostik. Für die Diagnostik ist es von großer Bedeutung, dass hochzylindrische Zellen gewonnen werden. Daher muss der Tupfer in den Zervikalkanal $(1-1,5 \mathrm{~cm}$ tief) oder in das Ostium urethrae eingeführt werden. Das so gewonnene Zellmaterial kann anschließend mittels Giemsa-Färbung analysiert werden. Hier sind im Falle einer Infektion dann sog. Einschlusskörperchen im Zytoplasma erkennbar.

Der Abstrich sollte mindestens $1 \mathrm{~cm}$ tief aus dem Zervikalkanal entnommen werden. 
In der Praxis ist der Antigennachweis von größerer Relevanz. Seit Juli 2007 ist dieser Nachweis Bestandteil der regulären Schwangerschaftsvorsorge und seit 2008 gibt es in Deutschland ein Nationales Screeningprogramm, in dem alle Frauen bis 25 Jahre $1 \times$ pro Jahr auf Chlamydien getestet werden sollen. Die Untersuchung erfolgt im Morgenurin, wobei hier die erste Urinportion von Bedeutung ist, da nur hier Zellmaterial nachweisbar ist. In dieser Urinprobe können die gruppenspezifischen Antigene mit monoklonalen Antikörpern nachgewiesen werden. Die Vermehrung der betreffenden Genabschnitte erfolgt entweder mit einer Polymerase-Kettenreaktion (PCR) oder Ligase-Kettenreaktion (LCR). Diese Verfahren weisen die höchste Sensitivität auf, da sie im Gegensatz zu den anderen Verfahren selbst geringe Bakterienmengen nachweisen; sie sind inzwischen die Standardtests in Deutschland. Die ersten Ergebnisse aus den Sentinel-Labortests im Rahmen des Screeningprogramms sind in Abb. $\mathbf{3}$ dargestellt.

Therapie. Aufgrund der Proliferation des Erregers in den Zylinderzellen der Zervixschleimhaut ist eine lokale vaginale Therapie nicht effektiv.

Indiziert ist eine systemische Therapie mit einer zielgerichteten Antibiose.
Aufgrund des intrazellulären Wachstums kommen Antibiotika zum Einsatz, die sich intrazellulär anreichern können. Hier empfiehlt sich die Anwendung von Tetrazyklinen über 10-14 Tage. Im Falle einer Infektion in der Schwangerschaft kommen Makrolide zur Anwendung, da Tetrazykline kontraindiziert sind. Bei eingeschränkter Compliance ist eine Einmaltherapie mit Azithromycin $1 \mathrm{~g}$ wirksam. Alternativ können Chinolone in einer Therapiedauer von 10-14 Tagen eingesetzt werden (s. auch Tab. 2). Es muss stets eine Partnermitbehandlung erfolgen, um den „Ping-Pong“Effekt zu vermeiden.

\section{Geburtshilfliche Aspekte}

Infektionen der schwangeren Frau mit C. trachomatis erhöhen das Risiko von Spontanaborten, Frühgeburten sowie für vorzeitigen Blasensprung. Letzterer kann durch Keimaszension zur peripartalen Transmission auf den Feten führen mit konsekutiver Inklusionskonjunktivitis und/oder Late-Onset-Pneumonie bei unbehandelten Patienten.

Schwangerschaft. Die Rate der Spontanaborte ist bei C.-trachomatis-Infektion in der Schwangerschaft erhöht. Die Ursachen für dieses Risiko werden kontrovers

Kasuistik zum fallorientierten Lernen

In der Notfallambulanz wird eine 19-jährige Patientin mit akut aufgetretenen, seit einer Woche persistierenden, zuletzt progredienten Unterbauchschmerzen vorstellig.

\section{Anamnese}

Es handelt sich um eine Nullipara mit letzter Periode vor 5 Tagen mit regelmäßigem Zyklus bei liegender Kupferspirale. Die Patientin lebt in einer festen Partnerschaft. Dyspareunie und Dysmenorrhö werden verneint. Es bestehen keine Vorerkrankungen und keine Voroperationen.

\section{Diagnostik}

Es zeigen sich ein negativer Schwangerschaftstest und eine Temperatur von $38,0^{\circ} \mathrm{C}$. In der Sonografie ergibt sich der V.a. eine Pysalpingx. Die Ovarien sowie der Uterus kommen unauffällig zur Darstellung. Es findet sich wenig freie Flüssigkeit im Douglas. Bei der vaginalen Untersuchung zeigt sich ein deutlicher Portioschiebeschmerz sowie Druckschmerz im gesamten Unterbauch, mäßiggradige Abwehrspannung und eingeschränkte Peristaltik. Die Portio erscheint entzündlich mit gelblichem Fluor. Mikrobiologische Abstriche werden entnommen. Laborchemisch zeigt sich eine Infektkonstellation mit erhöhtem CRP und Leukozyten.

\section{Therapie}

Da sich trotz Antibiose keine wesentliche Besserung der peritonitischen Symptomatik einstellt, wird am Tag nach der Aufnahme die Indikation zur diagnostischen Laparoskopie gestellt. Intraoperativ zeigt sich ein Adhäsionssitus mit Pyosalpinx beidseits. Nach Lösung der Tube vom Sigma entleert sich eine große Abzesshöhle. Es werden Abstriche (bakteriologisch und auf Chlamydien) sowie eine Spülzytologie entnommen. Im Ober- bauch zeigen sich die typischen Adhäsionen im Sinne eines Fitz-Hugh-Curtis-Syndroms. Die liegende Spirale wird entfernt. Es erfolgt eine ausgiebige Spülung und Adhäsiolyse. Postoperativ wird eine i.v. Antibiose mit Doxycyclin, Cephazolin und Metronidazol über 10 Tage eingeleitet.

\section{Diagnose}

Es wird die Diagnose einer PID bei Chlamydieninfektion gestellt. Dies bestätigt sich in der Histologie und im Abstrich.

\section{Postoperativer Verlauf}

Dieser gestaltet sich komplikationslos. Eine Partnermitbehandlung wird dringend empfohlen. Bei späterem Kinderwunsch und fehlendem Schwangerschaftseintritt sollte dringend eine diagnostische Laparoskopie mit Chromopertubation erfolgen. 
diskutiert. Eine bereits bei Konzeption vorliegende Endometritis mit erhöhten IgG-Antikörpern bei fehlendem Nachweis von C. trachomatis im Zervikalabstrich wurde zwar beobachtet, es gibt jedoch auch Hinweise, die gegen einen derartigen Zusammenhang sprechen.

Das Risiko der Frühgeburtlichkeit ist bei Infektion mit C. trachomatis um $50 \%$ erhöht.

Die kausalen Ursachen der Frühgeburtlichkeit bei Vorliegen einer Chlamydien-Infektion werden kontrovers diskutiert. Ob die alleinige Infektion der Schwangeren mit Chlamydien oder die deutlich erhöhte Prävalenz von Koinfektionen die Frühgeburt bedingt, ist unklar. Das Risiko einer bakteriellen Vaginose bei bestehender Chlamydien-Infektion ist z.B. doppelt so hoch im Vergleich zu Schwangeren ohne C.-trachomatis-Nachweis.

Infektion des Neugeborenen. Unbehandelt kommt es in $30-60 \%$ der Fälle zu einer perinatalen Übertragung der Chlamydien-Infektion auf das Neugeborene. Das Erscheinungsbild reicht von der sog. Inklusionskonjunk- tivitis bis hin zur Late-Onset-Pneumonie. Die Inklusionskonjunktivitis tritt in der Regel zwischen dem 5. und 15. Lebenstag, bei frühem vorzeitigen Blasensprung auch vor dem 5. Lebenstag auf. Bei Neugeborenen mit Konjunktivitis sollte man besonders aufmerksam bezüglich der möglichen Entstehung einer LateOnset-Pneumonie sein, denn diese stellt ein Frühwarnsymptom dar und ermöglicht es dem behandelnden Pädiater, die oft initial unerkannten Symptome einer Schwellung der nasalen Mukosa mit Mittelohrbeteiligung richtig zu interpretieren. Im weiteren Verlauf kommt es häufig zu anfallsartigem Husten, Tachypnoe, zum Teil auch zu Apnoen sowie zu Trinkschwäche und Gewichtsverlust. In der Diagnostik zeigt sich meist eine interstitielle Pneumonie.

Wochenbett. Bei bekannter Chlamydien-Infektion in der Schwangerschaft sollte ebenso das Risiko für die Entwicklung einer postpartalen Endomyometritis beachtet werden. Dies gilt ebenso auch nach Aborten und Abruptiones bei stattgehabter Chlamydien-Infektion.

\section{Kernaussagen}

\section{Mykosen}

Die Vulvovaginalkandidose (VVK) wird meist von Candida (C.) albicans verursacht und ist eine juckende Entzündung der Vagina, des Vestibulums und in schweren Fällen der gesamten Vulva mit allergoider, inflammatorischer Komponente bei Frauen unter Östrogeneinfluss und Immunschwäche. Juckreiz ist aber allein nur in etwa der Hälfte der Fälle Ausdruck einer VVK.

Die Diagnose wird durch Anamnese, klinische Zeichen und mikroskopischen Nachweis von Pseudohyphen gestellt. Blastosporen sind bei C. albicans nur die kolonisierende Variante, ihr Nachweis ohne klinisches Bild ist meist unbedeutend. Im Zweifel und bei Rezidiven muss eine Pilzkultur angelegt werden. Resistenztestungen sind nicht erforderlich.

Nur im 3. Trimenon einer Schwangerschaft sollte auch bei beschwerdefreier Patientin diese vaginale Kolonisation therapiert werden, um bei vaginaler Geburt die Kolonisation des Neugeborenen zu reduzieren, weil sonst häufig in der 2.-4. Lebens- woche „Mundsoor“ und „Windeldermatitis“ auftreten. Die vaginale Candida-Kolonisation und -Infektion steigert das Risiko für eine Frühgeburt, wenn auch nicht so stark wie Dysbiosen und bakterielle Vaginose.

Die Therapie der akuten VVK ist mit jedem auf dem Markt befindlichen vaginalen oder oralen Antimykotikum gleich gut möglich. Die chronisch rezidivierende VVK ist Folge einer Immunsuppression durch psychosozialen Stress, Genpolymorphismen, Atopie oder somatische Erkrankungen, z. B. eine diabetische Stoffwechsellage. Sie kann derzeit nur palliativ mit einer Langzeittherapie mit z. B. Fluconazol oral unterdrückt werden. Impfungen sind in Erprobung.

VVK durch Non-C.-albicans-Arten sind (noch?) mit insgesamt 10\% Anteil seltener. C. krusei ist resistent gegen orale Triazole. C. glabrata fast nicht sensibel gegen fast alle Antimykotika, sodass nicht dafür zugelassene teure Regimes mit z. B. Micafungin 14 Tage i. v. diskutiert werden.

\section{Chlamydien}

Chlamydien sind obligat intrazelluläre Bakterien, von denen die Arten C. trachomatis der Serogruppe A-C, C. trachomatis der Serogruppe D-K, die Serogruppen L1 - L3, C. pneumoniae und C. psittaci beim Menschen zu unterschiedlich ausgeprägten Infektionen führen können. Dabei gehört C. trachomatis der Gruppe D-K zu den häufigsten sexuell übertragenen bakteriellen Erregern. Die Infektion ist häufig symptomarm oder symptomlos (in 90\% der Fälle) und bleibt deshalb lange unerkannt. Sie führt zu Urethritis, Zervizitis, Endometritis und Salpingitis bzw. zu Manifestationen an den Augen und Gelenken. Infektionen bei Schwangeren erhöhen das Risiko für Spontanaborte und Frühgeburten. Durch peripartale Übertragung besteht für das Neugeborene das Risiko einer Inklusionskonjunktivitis bis hin zur Late-Onset-Pneumonie. Der Nachweis einer Infektion gelingt histologisch aus Zellmaterial oder molekularbiologisch mittels Antigennachweis. Lokale Therapien sind wirkungslos. Indiziert ist eine systemische Therapie mit gezielten Antibiotika, die sich intrazellulär anreichern können. 


\section{Über die Autoren}

\section{Werner Mendling}

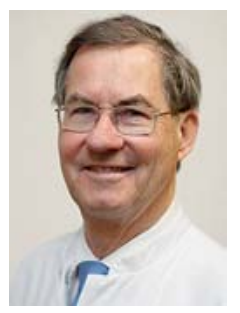

Prof. Dr. med. Jahrgang 1946. 19691975 Studium der Humanmedizin in Mainz und Düsseldorf. Promotion zum Dr. med. 1976-1995 Arzt, später Oberarzt an der Rheinischen Landesfrauenklinik und Hebammenlehranstalt Wuppertal-Elberfeld (seit 1985 durch Trägerwechsel Kliniken St. Antonius $\mathrm{gGmbH)}$. Habilitation über immunologische Untersuchungen bei Vaginalkandidosen an der Universität Witten/Herdecke. 1995-2000 Chefarzt der Klinik für Frauenheilkunde und Geburtshilfe (Perinatalzentrum) am Klinikum Frankfurt (Oder), 1995 Umhabilitation und Lehrbefugnis an der Humboldt-Universität (Charité) Berlin. 2000-2011 Chefarzt der Klinik für Frauenheilkunde und Geburtshilfe des Krankenhauses Am Urban Berlin, Trägerwechsel 2001 zu Vivantes, seitdem bis 2011 Chefarzt der Vivantes-Frauenkliniken Am Urban und im Friedrichshain. Seit 1987 Gründungs- und Vorstandsmitglied der Arbeitsgemeinschaft für Infektionen und Infektionsimmunologie (AGII) in der Deutschen Gesellschaft für Gynäkologie und Geburtshilfe. 2012 Gründung und Leitung des Deutschen Zentrums für Infektionen in Gynäkologie und Geburtshilfe an der St. Anna-Klinik in Wuppertal, 2014 und seit August 2015 komm. Chefarzt der St. Anna-Frauenklinik Wuppertal (seit April 2015 Helios Klinikum Wuppertal $\mathrm{GmbH}$ ). Wissenschaftlicher Schwerpunkt: Infektionen in Gynäkologie und Geburtshilfe.

\section{Monika Hampl}

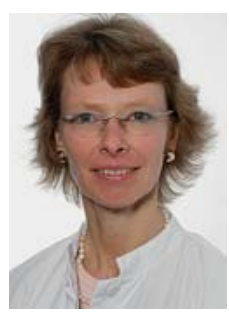

Prof. Dr. med. Jahrgang 1962. 1981 1987 Studium der Humanmedizin in Heidelberg. 1988-1989 Assistenzärztin an der Frauenklinik, St. Josephs-Hospital Wiesbaden. 1989-1994 Assistenzärztin an der Frauenklinik im Klinikum Mannheim der Universität Heidelberg. 1989 Promotion an der Universität Heidelberg. 1994-1998 Klinische Mitarbeiterin an der Klinik für Allgemein- und Thoraxchirurgie des Universitätsklinikums Dresden und wissenschaftliche Mitarbeiterin im molekulargenetischen Labor der Chirurgie. 1998-2000 von der Deutschen Krebshilfe (Mildred Scheel Stiftung) geförderter Forschungsaufenthalt an der Harvard University, Dana Farber Cancer Center, Boston, USA, mit Forschungsschwerpunkt: "Antiangiogenic gene therapy in gynecologic malignancies“. 2000-2001 Visiting Scientist am NIH (National Institutes of Health), National Cancer Institute, Bethesda, USA. Sept. 2001 - Jan. 2002 Fachärztin, Frauenklinik, Universitätsklini- kum Freiburg. Seit 2002 Oberärztin an der Frauenklinik, Heinrich-Heine-Universität Düsseldorf, dort 2004 Habilitation und Venia legendi in der Frauenheilkunde. 2007 Erwerb der Schwerpunktbezeichnung „Spezielle operative Gynäkologie“. Seit 2008 Leitende Oberärztin der Universitätsfrauenklinik Düsseldorf. Juli 2009 Verleihung einer außerordentlichen Professur an der Heinrich-Heine-Universität Düsseldorf. Seit 2014 Leiterin der ersten Zertifizierten Dysplasieeinheit in NRW.

\section{Interessenkonflikt}

WM hat in den vergangenen 3 Jahren Berater- und Vortragshonorare von folgenden Firmen erhalten: Abbott (Hannover), Aristo (Berlin), Dr. August Wolff (Bielefeld), Dr. Kade (Berlin), Johnson \& Johnson (Neuss), Medinova (Zürich), Pierre Fabre (Freiburg), Polichem (Lugano), Symbiopharm (Herborn). MH hat Referentenhonorare u.a. von Gedeon Richter (Köln), Abbott (Hannover), Sanofi Pasteur MSD (Berlin) und TEVA (Ulm) erhalten.

\section{Korrespondenzadresse}

Prof. Dr. med. Werner Mendling

Deutsches Zentrum für Infektionen in Gynäkologie

und Geburtshilfe

Monschaustraße 51

42369 Wuppertal

E-Mail: w.mendling@t-online.de

Prof. Dr. Monika Hampl

Leitende Oberärztin der Universitätsfrauenklinik Düsseldorf Leiterin des Dysplasiezentrums

Frauenklinik der Heinrich-Heine-Universität Düsseldorf

Moorenstraße 5

40225 Düsseldorf

E-Mail: hampl@med.uni-duesseldorf.de 


\section{Literatur}

1 Ginter-Hanselmayer G, Probst E, Weger W. Pubogenital tinea - an emerging „lifestyle-disease“? Mycoses 2013; 56 (Suppl 1): 2-3

2 AWMF; DGGG; Mendling W et al. Leitlinie Vulvovaginalkandidose - außer mukokutane Kandidose (AWMF 015-072) S2k. 2013. Im Internet: http://www.awmf.org/leitlinien/detail/Il/015-072.html; Stand: 26.10 .2015

3 Foxman B, Muraglia R, Dietz JP et al. Prevalence of recurrent vulvovaginal candidiasis in 5 European countries and the United States: results from an internet panel survey. J Low Genit Tract Dis 2013; 17: 340-345

4 Curran J, Hayward J, Sellers E et al. Severe vulvovaginitis as a presenting problem of type 2 diabetes in adolescent girls: a case series. Pediatrics 2011; 127: e1081-1085

5 Sobel JD. Vulvovaginal candidosis. Lancet 2007; 369: 1961-1971

6 Rylander E, Berglund AL, Krassny C et al. Vulvovaginal candida in a young sexually active population: prevalence and association with oro-genital sex and frequent pain at intercourse. Sex Transm Infect 2004; 80: 54-57

7 Neves NA, Carvallho LP, de Oliveira MA et al. Association between atopy and recurrent vaginal candidosis. Clin Exp Immunol 2005; 142: $167-171$

8 Witkin SS, Giraldo P, Linhares D. New insights into the immune pathogenesis of recurrent vulvovaginal candidiasis. Int J Gynecol Obestet 2000; 3: 114-118

9 Meyer $\mathrm{H}$, Göttlicher S, Mendling W. Stress as a cause of chronic recurrent vulvovaginal candidosis and the effectiveness of the conventional antimycotic therapy. Mycoses 2006; 48: 202-209

10 Blaschke-Hellmessen R. Subpartale Übertragung von Candida und ihre Konsequenzen. Vertical transmission of candida and its consequences. Mycoses 1998; 41 (Suppl. 2): 31-36

11 Yilmaz AE, Celik N, Soylu G et al. Comparison of clinical and microbial features of vulvovaginitis in prepubertal and pubertal girls. J Formos Med Assoc 2012; 111: 392-396

12 Mendling W. Vaginose, Vaginitis, Zervizitis und Salpingitis. 2. Aufl. Heidelberg: Springer; 2006

13 Weissenbacher T, Witkin SS, Ledger WJ et al. Relationship between clinical diagnosis of recurrent vulvovaginal candidiasis and detection of candida species by culture and polymerase chain reaction. Arch Gynecol Obstet 2009; 279: 125-129

14 Hettiarachchi N, Ashbee HR, Wilson JD. Prevalence and management of non-albicans vaginal candidiasis. Sex Transm Infect 2010; 86: 99-100

15 Mylonas I, Bergauer F. Diagnosis of vaginal discharge by wet mount microscopy: a simple and underrated method. Obstet Gynecol Surv 2011; 66: 359-368

16 Czeizel AE, Fladung B, Varga P. Preterm birth reduction after clotrimazole treatment during pregnancy. Eur J Obstet Gynecol Reprod Biol 2004; 116: 157-163

17 Kiss H, Petricevicz L, Husslein P. Prospective randomised controlled trial of an infection screening program to reduce the rate of preterm delivery. Br Med J 2004; 329: 371-375
18 Mendling W, Schlegelmilch R. Die 3-tägige Kombinationsbehandlung der Vulvovaginalkandidose mit Clotrimazol-200-mgVaginaltabletten und Clotrimazol-Creme für die Vulva ist signifikant besser als die mit Vaginaltabletten allein - eine ältere multizentrische, placebokontrollierte Doppelblindstudie. Geburtsh Frauenheilk 2014; 74: 355-360

19 Tietz HJ. Candida glabrata: Pathogenität und Stand der Dinge. Hautarzt 2012; 63: 868-871

20 Donders G, Bellen G, Byttebier G et al. Individualized decreasing dose maintenance fluconazole regimen for recurrent vulvovaginal candidiasis (ReCiDiF trial). Am J Obstet Gynecol 2008; 199 : 613.e1-613.e9

21 Rusch K, Schwiertz A. Candida autovaccination in the treatment of vulvovaginal Candida infections. Int J Gynecol Obstet 2007; 96: 130

22 Cassone A, Casadevall A. Recent progress in vaccines against fungal diseases. Curr Opin Microbiol 2012; 15: 427-433

23 Mendling W, Birkner V. Die Vakzination mit inaktivierten Laktobazillen oder Heliotherapie kann die Lebensqualität von Frauen mit chronisch rezidivierender vulvovaginaler Kandidose verbessern - Eine prospektive, 3-armige randomisierte Studie. Geburtsh Frauenheilk 2011; 71: 767-772

24 Martinez RC, Seney SL, Summers KL et al. Effect of Lactobacillus rhamnosus GR-1 and Lactobacillus reuteri RC-14 on the ability of Candida albicans to infect cells and induce inflammation. Microbiol Immunol 2009; 53: 487-495

25 Witt A, Kaufmann U, Bitschnau M et al. Monthly itraconazole versus classic homeopathy for the treatment of recurrent vulvovaginal candidiasis: a randomised trial. Br J Obstet Gynaecol 2009; 11; $1499-1505$

26 Hoffstetter SE, Barr S, LeFevre C et al. Self-reported yeast symptoms compared with clinical wet mount analysis and vaginal yeast culture in a specialty clinic setting. J Reprod Med 2008; 53: 402 406

27 Petersen EE. Infektionen in Gynäkologie und Geburtshilfe, Lehrbuch und Atlas. 5. Aufl. Stuttgart: Thieme; 2010

28 Fenton KA, Lowndes CM. Recent trends in the epidemiology of sexually transmitted infections in the European Union. Sex Transm Infect 2004; 80: 255-263

29 Bernstein KT, Marcus JL, Nieri G et al. Rectal gonorhea and chlamydia reinfection is associated with increased risk of HIV seroconversion. J Acquir Immune Defic Syndr 2010; 53: 537-543

$30 \mathrm{Ho}$ JL, He S, Hu A et al. Neutrophils from human immunodeficiency virus (HIV)-seronegative donors induce HIV replication from HIV-infected patients mononuclear cells and cell lines: an in vitro model of HIV transmission facilitated by Chlamydia trachomatis. J Exp Med 1995; 181: 1493-1505

31 Hiltune-Back E, Haikala O, Kautianinen $\mathrm{H}$ et al. Nationwide increase of Chlamydia trachomatis infection in Finnland. Highest rise among adolescent women and men. Sex Transm Dis 2003; 30: $737-741$

32 Robert Koch-Institut. Chlamydia trachomatis Laborsentinel; Ergebnisse 2008-2013. Epidemiolog Bull 2013; 46 


\section{CME-Fragen}

\section{CME•thieme.de}

cME-Teilnahme

- Viel Erfolg bei lhrer CME-Teilnahme unter http://cme.thieme.de

- Diese Fortbildungseinheit ist 12 Monate online für eine CME-Teilnahme verfügbar.

- Sollten Sie Fragen zur Online-Teilnahme haben, unter

http://cme.thieme.de/hilfe finden Sie eine ausführliche Anleitung.

1 Welche Aussage ist richtig? Die Vulvovaginalkandidose ...

A ist eine sexuell übertragbare Erkrankung.

B kommt in jedem Alter vor.

C tritt bevorzugt bei immunologischen Defiziten auf.

D wird nicht mit Imidazolpräparaten behandelt.

E wird häufig durch Candida glabrata verursacht.

2 Was sind typischerweise die anamnestischen Angaben und klinischen Zeichen bei Vulvovaginalkandidose?
A postmenstruelles Brennen und Rötung der Vulva
B prämenstruelles Jucken, veränderter Fluor und Rötung des Vestibulums
C periovulatorischer Fluor und genitaler Juckreiz
D postmenstruelles Jucken und gelb-grünlicher, dünner Fluor
E Juckreiz an den großen Labien und unangenehm riechender Fluor

3 Wie sind Vulvovaginalkandidose (VVK) und Vestibulodynie (VD) zu unterscheiden?
A Die VVK führt zu Jucken und Rötung im Vestibulum und mikroskopischem Nachweis von Blastosporen und Pseudohyphen, die VD zu Brennen und Schmerz im Vestibulum mit oder ohne Rötung bei Berührung.
B Bei VD findet man nie Blastosporen als Kolonisation.
C Die VVK kommt auch bei Mädchen in der hormonalen Ruhephase vor.
D Die VVK führt wie die VD zu Jucken und Brennen, der Candida-Nachweis ist entscheidend.
E Die VVK ist eine sexuell übertragbare Erkrankung, die VD nicht.

$4 \quad$ Wie wird die akute Candida-albicans-Vulvovaginalkandidose therapiert?
A 1-Tages-Therapie mit Metronidazol oral
B Clotrimazol vaginal je nach Dosierung als 1-, 3- oder 6-Tage-Therapie ohne Partnerbehandlung
C Clotrimazol vaginal je nach Dosierung als 1-, 3- oder 6-Tage-Therapie mit Partnerbehandlung
D Nystatin oral
E Fluconazol-Kapseln 200 mg nach dem Schema von Donders in der Leitlinie Vulvovaginalkandidose

5 Welche Arten von Pilzen sind aus gynäkologischer Sicht klinisch von Bedeutung für Vulvovaginalkandidosen?
A Candida (C.) albicans, C. glabrata und Saccharomyces cerevisiae
B C. albicans, C. tropicalis und Dermatophyten
C C. albicans, C. krusei und Schimmelpilze
D C. albicans, C. glabrata und C. krusei
E C. albicans, C. krusei und C. kefyr 


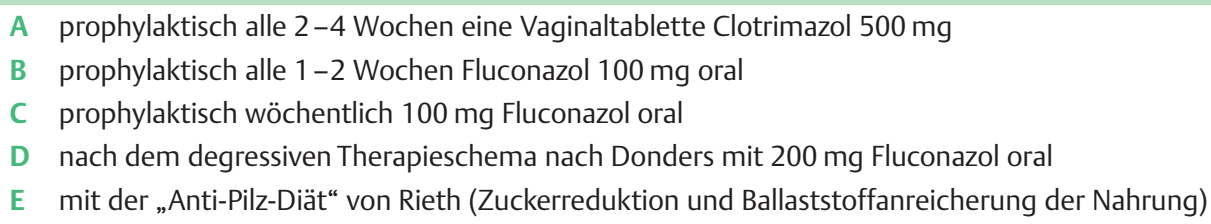

\section{Welche Aussage ist falsch?}

A Chlamydien sind obligat intrazellulär wachsende Bakterien.

B Chlamydien kommen als Elementarkörperchen und als Retikularkörperchen vor.

C In der Vermehrungsphase können die Chlamydien gut mit Antibiotika behandelt werden.

D In der Vermehrungsphase liegen die Erreger als Elementarkörperchen vor.

E Ein vollständiger Vermehrungszyklus von Chlamydien in einer Wirtszelle dauert 36-50 Stunden.

\section{Welche Aussage trifft nicht zu?}
A Chlamydien der Serogruppe A-C verursachen Augenentzündungen (Trachom).
B Chlamydien der Serogruppe L1 - L3 verursachen das Lymphogranuloma venereum.
C Chlamydien sind hochkontagiös und werden nie von Tier auf Mensch übertragen.
D Die Therapie der chlamydienbedingten chronischen Arthritis muss über 30-90 Tage erfolgen.
E C. pneumoniae verursacht Lungenentzündung.

9 Welche Aussage ist falsch? Die Chlamydien-Infektion bei der Frau ...
A verursacht zunächst eine Zervizitis und Urethritis.
B kann aszendieren und zur Adnexitis führen.
C ist eine häufige Ursache des „PID“-Syndroms.
D wird in den meisten Fällen direkt erkannt und behandelt.
E ist eine häufige Ursache der Unfruchtbarkeit.

\section{Welche Aussage trifft zu?}
A Chlamydien sind immer resistent gegen Antibiotika.
B Die Therapie der Wahl ist Aciclovir.
C Männer sind nie von dem Erreger betroffen.
D Chlamydien-Infektionen sind immer asymptomatisch.
E Chlamydien können peripartal auf das Neugeborene übertragen werden. 